\title{
Characteristics of daily life gait in fall and non fall-prone stroke survivors and controls
}

\author{
Michiel Punt ${ }^{*}$, Sjoerd M. Bruijn ${ }^{2,3}$, Kimberley S. van Schooten ${ }^{2}$, Mirjam Pijnappels ${ }^{2}$, Ingrid G. van de Port ${ }^{4}$, \\ Harriet Wittink' and Jaap H. van Dieën ${ }^{2}$
}

\begin{abstract}
Background: Falls in stroke survivors can lead to serious injuries and medical costs. Fall risk in older adults can be predicted based on gait characteristics measured in daily life. Given the different gait patterns that stroke survivors exhibit it is unclear whether a similar fall-prediction model could be used in this group. Therefore the main purpose of this study was to examine whether fall-prediction models that have been used in older adults can also be used in a population of stroke survivors, or if modifications are needed, either in the cut-off values of such models, or in the gait characteristics of interest.

Methods: This study investigated gait characteristics by assessing accelerations of the lower back measured during seven consecutive days in 31 non fall-prone stroke survivors, 25 fall-prone stroke survivors, 20 neurologically intact fall-prone older adults and 30 non fall-prone older adults. We created a binary logistic regression model to assess the ability of predicting falls for each gait characteristic. We included health status and the interaction between health status (stroke survivors versus older adults) and gait characteristic in the model.

Results: We found four significant interactions between gait characteristics and health status. Furthermore we found another four gait characteristics that had similar predictive capacity in both stroke survivors and older adults.

Conclusion: The interactions between gait characteristics and health status indicate that gait characteristics are differently associated with fall history between stroke survivors and older adults. Thus specific models are needed to predict fall risk in stroke survivors.
\end{abstract}

Keywords: Gait quality, Gait quantity, Accelerometry, Stability, Accidental falls, Fall risk

\section{Background}

Falls are a major problem in the growing older population. Falls can result in serious injuries leading to considerable medical costs [1]. In stroke survivors, fall rates are higher in comparison to healthy older adults $[2,3]$. Falls may increase the fear of falling and may subsequently reduce physical activity [4], which can result in physical deconditioning and may further increase fall risk in the long term.

Objective fall risk assessment often involves assessment of balance control, for example with the Berg Balance Scale [5]. However, the relation between deficits in balance control and fall rates in stroke survivors is

\footnotetext{
* Correspondence: Michiel.punt@hu.nl

${ }^{1}$ Research group Lifestyle and Health, Utrecht University of Applied Sciences, Bolognalaan 101, Utrecht 3584 JW, The Netherlands

Full list of author information is available at the end of the article
}

inconsistent $[3,5]$. It has been suggested that this might be due to fact that most balance tests are static in nature [6], while most falls occur during dynamic tasks such as walking and transfers [3]. Interestingly, several characteristics of gait quality have been shown to differentiate fallers from non-fallers among older adults [7-9]. These characteristics can be measured in daily life by accelerometry, and reflect aspects such as stability, symmetry, smoothness and variability. van Schooten et al. [9]. demonstrated the added value of such gait characteristics to conventional clinical predictors of fall risk.

A similar approach may be useful for stroke survivors, and could add value to existing clinical tests. However, quantity and quality of gait are different in stroke survivors [10-12] than in healthy individuals, and it is therefore unclear whether a fall risk prediction model as used by van Schooten et al. [9] can be used in stroke 
survivors. For example, stroke survivors have a more asymmetrical [11] and unstable gait [10] compared to age matched controls. Furthermore stroke survivors are physically less active [12] and physical activity has been associated to falls as well [9]. Thus, even if the same gait quality characteristics predict falling in stroke survivors and in healthy controls, it may be that regression coefficients for these characteristics are markedly different in a prediction model for stroke survivors compared to models developed for healthy older adults.

To this date, exploring the potential of fall prediction models based on gait characteristics has been limited to older adults; however, gait in stroke survivors is remarkably different and fall incidences are a frequent problem in stroke survivors. To explore the potential of using daily life gait assessment to predict falls in stroke survivors, the main purpose of the current study was to examine whether fall-prediction models that have been used in healthy older adults [7-9] can also be used in a population of stroke survivors, or if modifications are needed, either in the regression coefficients of such models, or in the gait characteristics of interest.

\section{Methods \\ Participants}

We tested community-dwelling stroke survivors as well as healthy older adults. Stroke survivors were recruited via local physical therapy centers and through national peer group meetings. Stroke survivors were above the age of 18 , at least 1 year post stroke and were living in the community independently. Stroke survivors were excluded from the study if they had a functional ambulation category of two or less. Data for the healthy older adults group were derived from a data set of a larger study on 'fall risk assessment in older adults' (FARAO) $[8,9]$. We only included participants from who we were certain that they were free of any neurological damage, such as a history of stroke or Parkinson's disease. Control participants for this study were recruited via general practitioners, pharmacies and residential care facilities in the area of Amsterdam, The Netherlands. Participants were excluded from the study if they had severe cognitive disorders, as indicated by a minimal mental state examination score of 24 or less [13]. All participants were able to walk independently for at least $20 \mathrm{~m}$, if necessary with a walking aid. The research protocol was approved by the medical ethical committees of the University Medical Center Utrecht and the VU medical center Amsterdam, The Netherlands. All participants signed informed consent and treatment of the participants was according to Good Clinical Practice.

\section{Data collection}

Fall status was determined using a self-reported questionnaire asking about falls in the last 12 months prior to determining gait characteristics. A fall was defined as; 'any unanticipated event that results in participant coming to the ground, floor or lower level' [14]. To estimate quantitative and qualitative gait characteristics, participants were asked to wear a tri-axial accelerometer (55 g), (McRoberts, Den Haag, The Netherlands) at the middle of the lower back using an elastic belt [15]. The accelerometer was aligned to coincide with the anteriorposterior (AP), medio-lateral (ML) and vertical (VT) body-axis. Participants were instructed to realign the accelerometer during the monitoring period if necessary. Data were sampled at 100 samples/s with a range $\pm 6 \mathrm{~g}$ and digitally stored on a mini SD card. Participants were instructed to wear the accelerometer for seven consecutive days, preferably during day and night, but were allowed to take it off when going to bed. The accelerometer was removed during showering and other waterrelated activities to prevent damage.

\section{Data analysis}

Based on health status and fall history of at least one fall in the previous year, we classified participants into four groups: non fall-prone stroke survivors (NF-SS), fallprone stroke survivors (F-SS) and a control (CON) group of older adults thus fall-prone older adults (F$\mathrm{CON})$ and non fall-prone older adults (NF-CON) and used these groups for further analyses.

Gait activity was identified from the weekly time series using a validated algorithm for gait detection and gait quantification [16]. The algorithm searches for gait activity based on the spectral content of the AP acceleration and discards periods of activity shorter than $8 \mathrm{~s}$. Total monitoring time was defined as the time between the first and last gait episode of each participant over the 7 days. Prior to estimating gait characteristics, potential accelerometer misalignment was corrected according to the method described by Rispens et al. [15].

Gait quantity was expressed as the duration of gait activity per $24 \mathrm{~h}$ and the number of walking bouts per $24 \mathrm{~h}$. We classified walking bouts of $24 \mathrm{~s}$ or shorter as short walking bouts. Short walking bouts are likely to be executed predominantly in at-home settings, which might affect gait characteristics (for instance, turning affects step length symmetry, and usually in-home walking coincides with more turning as compared to walking outdoors). All walking bouts were divided into 8-s epochs for further analysis. We calculated the number of $8 \mathrm{~s}$ epochs that were part of the short walking bouts and divided this by the total number of epochs. Thus we were able to determine whether the characteristics of 
gait quality were derived under similar circumstances between groups.

We estimated gait quality characteristics that have shown promise in differentiating between fallers and non-fallers among older adults [8]. Specifically, we calculated gait speed [17], gait symmetry determined by the harmonic ratio (HR) [18]. This HR measure divides the sum of the first ten even harmonics through the sum of the first ten odd harmonics. Symmetrical gait in the VT and AP direction will predominantly contain even harmonics which will result in a higher HR. The smoothness of gait was determined by dividing the ground frequency (first harmonic) of the time series by the first six harmonics of the time series, the index of harmonicity (IH) [19]. A complete smooth gait can be described by one sinusoidal function and no higher harmonics would be necessary to describe the signal. Subsequently this would result in a higher $\mathrm{IH}$ value. Several indicators of gait variability were determined. Firstly the amplitude of the dominant peak which represents the 'strength' of the dominant peak relative to the rest of the signal [20], and hence a high value represents a low variability. Secondly the width of peak of the power spectrum reflects the dispersion of the dominant peak [20] and hence a higher value represents a higher variability. Thirdly stride frequency variability, and fourthly local dynamic stability expressed as the local divergence exponent (LDE), which quantifies the exponential rate of divergence from initially nearby kinematics states as a function of stride time [8]. A higher LDE indicates a faster diverging acceleration signal and indicates a more unstable gait pattern. Except for gait speed and stride time all these characteristics were determined in three directions using algorithms previously described by Rispens et al. [8]. Estimation of gait quality characteristics was performed on each epoch of 8-s length, which was sufficient long for estimating spectral features [21]. For each characteristic, the median value over all gait epochs of a participant was used for statistical evaluation. We took the median value as the median is less sensitive in comparison to the mean for outliers in the estimated gait characteristic.

\section{Statistics}

For each group, means and standard deviations are reported. Participant characteristics and monitoring duration were compared between groups using health status (stroke survivor or healthy older adult) and fall history as two categorical factors in an analysis of variance (ANOVA). When these analyses revealed differences between groups, the variable concerned was used as a covariate to control for its effects in subsequent analyses.

To facilitate objective comparison between independent variables we $\mathrm{z}$ transformed continues variables prior to performing the logistic regression. We developed a fall prediction model per gait characteristic using binary logistic regression. Fall history was used as dependent variable, while the gait characteristic and the categorical variable health status (stroke survivors versus healthy older adults, coded as 1 and 0 respectively) were the independent variables. The interaction between health status and the gait characteristic was also included in the model, but if the interaction did not reach a $p$-value of $\leq 0.05$ it was removed from the model and a new model with health status and the gait characteristic only was created. The odds ratio (OR) is a number indicating the amount of increased fall risk per unit increase of the independent variable. If a significant $O R$ is found for a specific gait characteristic only, this implies that it is associated with fall history and that this association does not depend on health status (controls, older adults and stroke survivors). If in addition a significant OR is found for health status, but no interaction, this implies that fall risk is dependent on health status, but that the change in risk with a unit change in the gait characteristic is independent of health status. If an interaction effect is found, this implies that the relation of the gait characteristic with fall history is different between health status groups, suggesting that a specific model is needed for stroke survivors. All statistical analyses were performed using SPSS software version 20.0, and a $p$-value of $\leq 0.05$ was considered statistically significant.

\section{Results}

A total of 106 participants volunteered for the study. Of the 56 participating stroke survivors 25 (45\%) had experienced at least one fall in the previous year. A total of 50 control older adults participated of whom 20 (40\%) had experienced at least one fall in the previous year. Participant characteristics and monitoring duration results for each group are presented in Table 1. Tables 2 and 3 provide an overview of which gait characteristics show promise in regard to predicting fall risk in stroke survivors and older adults. More precise, Table 2 provides an overview of quantitative and qualitative gait characteristic values between stroke survivors and older adults and is as well subdivided in fallers and non fallers. An overview of the corresponding Odds ratios (OR) between all four groups and $p$-values derived from the binary logistic regression models are presented in Table 3.

Two-way ANOVAs for participant characteristics revealed no differences in monitoring duration and anthropometrics between groups, but showed a significant interaction effect on age, with the NF-SS group being significantly younger than the other three groups. Further results were corrected for this difference by using age as covariate. 
Table 1 Participant characteristics for the four groups

\begin{tabular}{|c|c|c|c|c|c|c|c|c|c|c|}
\hline & \multirow{2}{*}{$\begin{array}{l}\text { NF-SS } \\
\text { Mean } \pm \text { SD }\end{array}$} & \multirow{2}{*}{$\begin{array}{l}\text { F-SS } \\
\text { Mean } \pm S D\end{array}$} & \multirow{2}{*}{$\begin{array}{l}\text { NF-CON } \\
\text { Mean } \pm S D\end{array}$} & \multirow{2}{*}{$\begin{array}{l}\text { F-CON } \\
\text { Mean } \pm S D\end{array}$} & \multicolumn{2}{|c|}{ Health status } & \multicolumn{2}{|c|}{ Fall history } & \multicolumn{2}{|c|}{ Interaction } \\
\hline & & & & & $\mathrm{F}$ & $P$-value & $\mathrm{F}$ & $P$-value & $\mathrm{F}$ & $P$-value \\
\hline Female/male & $15 / 16$ & $15 / 10$ & $13 / 17$ & $14 / 6$ & & & & & & \\
\hline Age (years) & $64.1 \pm 11.6$ & $69.0 \pm 9.2$ & $71.9 \pm 4.1$ & $74.9 \pm 8$ & 14.98 & $<0.001$ & 0.29 & 0.59 & 5.21 & 0.024 \\
\hline Height (m) & $171.4 \pm 8.8$ & $172.3 \pm 9.3$ & $169.4 \pm 9.2$ & $170.4 \pm 7.7$ & 1.37 & 0.244 & 0.01 & 0.99 & 0.33 & 0.564 \\
\hline Weight (kg) & $79.7 \pm 14.7$ & $82.9 \pm 16.5$ & $75.1 \pm 10.5$ & $75.3 \pm 13.7$ & 3.87 & 0.051 & 3.89 & 0.534 & 0.34 & 0.561 \\
\hline BMI (kg/m2) & $27.0 \pm 3.8$ & $28.1 \pm 6.5$ & $25.8 \pm 2.5$ & $26.1 \pm 3.7$ & 3.52 & 0.063 & 0.71 & 0.400 & 0.21 & 0.645 \\
\hline Monitoring(days) & $6.5 \pm 0.5$ & $6.5 \pm 0.4$ & $6.4 \pm 0.7$ & $6.5 \pm 0.6$ & 0.85 & 0.771 & 0.48 & 0.827 & 0.945 & 0.333 \\
\hline
\end{tabular}

Main effects for health status and fall history and their interaction are presented. Significant $p$-values are printed in bold

NF-SS non-fallers, stroke survivors, F-SS faller, stroke survivor, NF-CON non-faller control group of older adults, F-CON faller control group of older adult

Table 2 Quantitative and qualitative gait characteristics for the four groups

\begin{tabular}{|c|c|c|c|c|}
\hline & NF-SS & F-SS & NF-CON & $\mathrm{F}-\mathrm{CON}$ \\
\hline Quantitative measures & Mean \pm SD & Mean \pm SD & Mean \pm SD & Mean \pm SD \\
\hline Gait activ & $35.9 \pm 20.1$ & $34.4 \pm 24$ & $47.5 \pm 21.8$ & $50 \pm 19.8$ \\
\hline B/day & $127 \pm 64.5$ & $125 \pm 77.3$ & $158 \pm 54.4$ & $152 \pm 49.9$ \\
\hline hort & $91.6 \pm 4.8$ & $92.4 \pm 6.2$ & $89.7 \pm 6$ & $90.2 \pm 3.9$ \\
\hline & & & & \\
\hline \multicolumn{5}{|l|}{ Qualitative measures } \\
\hline ait cneed $(\mathrm{m} / \mathrm{c})$ & & & $0.93 \pm 0.30$ & $0.90 \pm 0.21$ \\
\hline ride t & $1.26 \pm 0.20$ & $1.44 \pm 0.40$ & $1.14 \pm 0.13$ & \\
\hline VT & & & & \\
\hline $\mathrm{ML}$ & & $1.35 \pm$ & & \\
\hline larmonic Ratio AP & $1.17 \pm 0.20$ & $1.07 \pm$ & $1.71=$ & $1.39=$ \\
\hline eq. & & & & \\
\hline eq. Variabili & & $0.21 \pm 0.05$ & & $0.17 \pm 0.04$ \\
\hline eq. vain & $0.17 \pm 0.04$ & $0.19 \pm 0.05$ & & \\
\hline VT & & & & \\
\hline IVIL & $0.38 \pm 0.21$ & $0.50 \pm 0.25$ & $0.34 \pm$ & \\
\hline AP & & & & \\
\hline mplitude (psd) VT & $0.47 \pm 0.12$ & $0.40 \pm 0.09$ & $0.56 \pm 0.09$ & $0.58 \pm 0.11$ \\
\hline mplitude ( & $0.40 \pm 0.15$ & $0.50 \pm 0.19$ & $0.36 \pm$ & 0.36 \\
\hline Implitude (psd) AP & $0.42 \pm 0.11$ & $0.46 \pm 0.17$ & $0.42 \pm 0.09$ & $0.41=$ \\
\hline Width (psd) VT & $0.98 \pm 0.01$ & $0.99 \pm 0.02$ & $0.95 \pm 0.01$ & $0.94 \pm 0.01$ \\
\hline Vidth (psd) ML & $0.95 \pm 0.02$ & $0.95 \pm 0.03$ & & \\
\hline 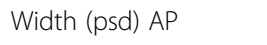 & $0.95 \pm 0.02$ & $0.95 \pm 0.01$ & $0.94 \pm 0.01$ & \\
\hline LDE/stride VT & $0.98 \pm 0.19$ & $1.02 \pm 0.19$ & $0.92 \pm 0.15$ & $0.94 \pm 0.16$ \\
\hline LDE/stride ML & $0.89 \pm 0.18$ & $0.90 \pm 0.20$ & $0.71 \pm 0.08$ & $0.78 \pm 0.11$ \\
\hline DE/stride AP & $0.87 \pm 0.19$ & $0.90 \pm 0.21$ & $0.72 \pm 0.10$ & $0.78 \pm 0.11$ \\
\hline \multicolumn{5}{|c|}{$\begin{array}{l}\text { able 2, Quantitative and qualitative gait characteristics for all four groups } \\
\text { expressed as means and standard deviations. Abbreviations: WB is walking } \\
\text { oouts, Freq. Variability is the stride frequency variability, IH is the Index of } \\
\text { tarmonicity, Amplitude (psd) is the amplitude of the dominant peak in the } \\
\text { oower spectral density, Width (psd) is the width of the dominant peak in the } \\
\text { oower spectral density, LDE/stride is the local divergence exponent per stride. } \\
/ T \text { is the vertical direction, ML is the medio-lateral direction and AP is the } \\
\text { aterior-posterior direction. Significant associations derived from logic regression } \\
\text { Table 3) are printed in bold }\end{array}$} \\
\hline
\end{tabular}

No associations with a history of falls were found for gait quantity characteristics (see Table 3). Four gait quality characteristics were found to be associated with a history of falls independent of health status (see Table 3). A reduced gait symmetry (HR) in the VT and AP direction and

Table 3 Binary logistic regression

\begin{tabular}{|c|c|c|c|}
\hline & Gait characteristic & Health status & Interaction \\
\hline Quantitative measures & $\mathrm{OR}(p)$ & $\mathrm{OR}(p)$ & $\mathrm{OR}(p)$ \\
\hline Gait activity (min/day) & $1.01(.94)$ & $0.82(.63)$ & \\
\hline WB/day & $0.94(.74)$ & $0.85(.69)$ & \\
\hline Short WB (\%) & $1.17(.43)$ & $0.88(.76)$ & \\
\hline Short WB epochs(\%) & $1.48(.06)$ & $0.99(.99)$ & \\
\hline \multicolumn{4}{|l|}{ Qualitative measures } \\
\hline Gait speed (m/s) & $0.64(.06)$ & $1.17(.71)$ & \\
\hline Stride time (seconds) & $1.71(.07)$ & $1.19(.68)$ & \\
\hline Harmonic Ratio VT & $0.54(.02)$ & $1.61(.33)$ & \\
\hline Harmonic Ratio ML & $1.23(.31)$ & $0.71(.41)$ & \\
\hline Harmonic Ratio AP & $0.34(<.01)$ & $2.72(.06)$ & \\
\hline Freq. Variability VT & $0.81(.34)$ & $0.81(.59)$ & \\
\hline Freq. Variability ML & $1.45(.08)$ & $1.01(.94)$ & \\
\hline Freq. Variability AP & $1.41(.12)$ & $1.08(.86)$ & \\
\hline $\mathrm{IHVT}$ & $0.46(<.01)$ & $1.81(.23)$ & \\
\hline $\mathrm{IH} \mathrm{ML}$ & $1.64(.05)$ & $0.65(.37)$ & $0.22(<.01)$ \\
\hline $\mathrm{HAP}$ & $0.84(.40)$ & $0.87(.73)$ & \\
\hline Amplitude (psd) VT & $0.44(.02)$ & $1.21(.70)$ & $2.76(.04)$ \\
\hline Amplitude (psd) ML & $1.54(.04)$ & $1.05(.91)$ & \\
\hline Amplitude (psd) AP & $1.04(.83)$ & $0.90(.81)$ & \\
\hline Width (psd) VT & $1.11(.63)$ & $0.09(.04)$ & $0.01(.02)$ \\
\hline Width (psd) ML & $0.98(.94)$ & $0.82(.62)$ & \\
\hline Width (psd) AP & $0.87(.51)$ & $0.81(.61)$ & \\
\hline LDE/stride VT & $1.31(.21)$ & $1.02(.97)$ & \\
\hline LDE/stride ML & $1.16(.54)$ & $2.23(.13)$ & $4.86(.03)$ \\
\hline LDE/stride AP & $1.54(.06)$ & $1.31(.55)$ & \\
\hline
\end{tabular}

Quantitative and qualitative gait characteristics association with a history of falls. Health status includes stroke survivors versus older adults. Data was z-transformed prior to logistic regression. Significant associations are printed in bold 
decreased gait smoothness (IH) in the VT direction were associated with a history of falls in both groups. Moreover an increase in the dominant amplitude of the power spectrum in the ML direction was associated with an increased fall risk in both groups. Increased stride time and reduced gait speed showed a trend of increased fall risk in both groups, respectively ( $p=.06$ and $p=.07$ ).

In addition for four gait quality characteristics a significant interaction term between health status and gait characteristic, was predictive for fall history (see Table 3 ). For gait smoothness (IH) in the ML direction, this indicated that a higher $\mathrm{IH}$ increased fall risk, but less so in stroke patients, although stroke increased the fall risk. Moreover an larger width of the dominant peak of the power spectrum in VT direction higher fall risk in stroke survivors but lower in older adults. A higher amplitude of the dominant peak in VT was associated with a lower fall risk in stroke survivors but not in older adults, with stroke increasing fall risk as well. Finally, an increase in the local divergence exponent (decrease in local dynamic stability) in the ML direction, increased fall risk in stroke survivors, but not in healthy older adults.

\section{Discussion}

The main purpose of the study was to test whether fallprediction models that have been used in healthy older adults can also be used in a population of stroke survivors, or if modifications are needed, either in the regression coefficients, or in the gait characteristics of interest.

Previous studies assessing gait in older adults have shown that gait speed [22], gait variability [23], local dynamic stability $[8,9,24]$ and symmetry [25] provide valuable information concerning fall risk in older adults. Our results are partly in line with these findings as we found several similar gait characteristics that were able to predict falls in both groups. However the limited number of participants in our study reduced statistical power in comparison to previous work [8,9], which could explain that not all findings are reproduced in this study. For instance gait speed and stride time showed only a trend of predictive ability in both groups, respectively $(p=.06$ and $p=.07)$.

Interestingly, we found four interactions indicating a different relation between gait quality and fall history in stroke survivors compared to the group of healthy older adults. In addition gait symmetry in the AP direction was predictive for falls in both groups, but health status showed a trend $(p=.06)$ indicating a different cut off value in the regression. Thus, since we found gait characteristics that were predictive for falls in both groups but we also found gait characteristics with a interaction or different cut off value the overall results indicate that predicting falls in stroke survivors based on daily-life gait characteristics is possible but requires a stroke specific fall-prediction model.

Surprising results were found for the index of harmonicity, which is calculated by dividing the power of the fundamental frequency by the power of the first six harmonics. This measure is thought to reflect the smoothness of gait. Interestingly, based on mean and standard deviations between groups the F-SS group had the highest index of harmonicity in the ML direction but the lowest in the AP and VT directions. This different relation between ML and AP, VT direction for the index of harmonicity can possibly be explained by a more pronounced and rapid shift of the center of mass between both legs in this group, to reduce the time standing on the paretic leg. Such fast movement results in a high peak in the acceleration signal at the stride frequency. Therefore the index of harmonicity in the ML direction, measured at the pelvis might reflect more a rigid gait pattern and loss of control in the paretic leg rather than smoothness of gait. Considering the present differences between groups, it is a gait characteristic of interest in the stroke population. Moreover, this opposite relation between ML and AP, VT direction for the index of harmonicity was confirmed by our findings on the amplitude of the dominant peak in the power spectrum. The amplitude of the dominant peak was highest for the F-SS group in the ML direction but lowest in the VT direction, which is in line with the findings by Weiss et al. [7].

Although none of the gait quantity characteristics were associated with a history of falls, gait activity and the number of walking bouts seems to be reduced in stroke survivors, see also Table 2. Since most falls occur during gait [26], this reduced gait activity could be a fall risk avoidance strategy. However reduced gait activity may cause further deconditioning and subsequently increased fall risk in the long-term.

\section{Study limitations}

To divide our groups of interest into fallers and nonfallers we have used self-reported retrospective fall incidents. Retrospective fall reports can be influenced by recall bias and their relation with gait quality may slightly differ from prospective fall reports [9]. These differences may have influenced our classification. Second, the identification of gait epochs for estimating gait characteristics was accomplished by a gait detection algorithm [16]. Although validity and reliability was good for slow and fast walking, it still remains unknown to what extent the algorithm identified other forms of cyclic movements such as biking. Misclassifications of gait activity or for instance wearing the accelerometer away from the midline of the lower back will result in deviating estimations of gait characteristics for those epochs. 
Yet the error in our final estimating gait characteristics is limited by taking the median value over all epochs rather than the mean. Third, the results showed differences in percentages of short walking bouts between groups. This suggests that the median value for the qualitative gait characteristics were estimated based on slightly different environmental circumstances. This is an important finding, because for example gait symmetry may be affected by bends and shorter walking bouts are probably performed in a more complex setting, which contains more bends. To examine whether this finding influenced our results we compared gait characteristics between groups including only walking bouts lasting $16 \mathrm{~s}$ or more. Mean values were somewhat different but no consistent changes were found and the main findings would have been the same as presented here. In addition we reanalyzed our statistical models taking weight and BMI as covariates, since both variables were nearly significant different between groups. OR were slightly different yet the same interactions were still present.

\section{Conclusion}

In conclusion, due to the present interactions found, several gait characteristics are differently associated with a history of falls in stroke survivors as in older adults. This suggests that specific models are needed to predict fall risk in stroke survivors.

\section{Abbreviations \\ AP, anterior-posterior; BMl, body mass index; F-CON, fall prone controls; F-SS, fall-prone stroke survivors; $H R$, harmonic ratio; $I H$, index of harmonicity; $L D E$, local divergence exponent; $\mathrm{ML}$, medio-lateral; $\mathrm{NF}-\mathrm{CON}$, non fall prone controls; NF-SS, non fall-prone stroke survivors; OR, odds ratio; VT, vertical}

\section{Acknowledgement}

The authors would like to thank all participants.

\section{Funding}

Michiel Punt was supported by a grant from the Netherlands organization for Scientific Research (NWO \#023-003-141). Sjoerd M. Bruijn was supported by a grant from the Netherlands Organization for Scientific Research (NWO \#451-12-041).

\section{Availability of data and materials}

Average values per group and dispersion values per group from which statistical analysis are based are provided in the manuscript.

\section{Authors' contributions}

MP has made substantial contribution in the experimental design, data collection, data analysis and drafting the manuscript. SMB has made substantial contribution in the experimental design, statistics and revising the manuscript. KS has made substantial contribution in data collection, data analysis and revising the manuscript. MPI has made contributions in setting up the experiment and revising the manuscript. IP had made substantial contribution in facilitating the research project and revising the manuscript. HW has made substantial contribution in facilitating the research and revising the manuscript. JD has made a substantial contribution in revising the manuscript. All authors read and approved the final manuscript.

\section{Competing interests}

Kimberley S van Schooten was partially supported by a commercial grant from McRoberts (the Hague, the Netherlands) who had no influence in the execution, analysis or writing of this manuscript.

\section{Ethics approval and consent to participate}

All participants gave written informed consent and medical and ethical approval was provided by the medical \& ethical commission 'Noord Brabant', the Netherlands, study registered by: NL4912602814.

\section{Author details}

'Research group Lifestyle and Health, Utrecht University of Applied Sciences, Bolognalaan 101, Utrecht 3584 JW, The Netherlands. ${ }^{2}$ Move Research Institute Amsterdam, Department of Human Movement Sciences, Vrije Universiteit Amsterdam, Amsterdam, The Netherlands. ${ }^{3}$ Department of Orthopedics, first affiliated Hospital of Fujian Medical University, Fuzhou, Fujian, People's Republic of China. ${ }^{4}$ Revant Rehabilitation Center Breda, Breda, The Netherlands.

Received: 9 December 2015 Accepted: 17 July 2016

Published online: 27 July 2016

\section{References}

1. Roudsari BS, Ebel BE, Corso PS, Molinari N-AM, Koepsell TD. The acute medical care costs of fall-related injuries among the U.S. older adults. Injury. 2005;36:1316-22.

2. Deandrea S, Lucenteforte E, Bravi F, Foschi R, La Vecchia C, Negri E. Risk factors for falls in community-dwelling older people: a systematic review and meta-analysis. Epidemiology. 2010;21:658-68.

3. Weerdesteyn V, De Niet M, Van Duijnhoven HJR, Geurts ACH. Falls in individuals with stroke. J Rehabil Res Dev. 2008;45:1195-213.

4. Jefferis BJ, lliffe S, Kendrick D, Kerse N, Trost S, Lennon LT, Ash S, Sartini C, Morris RW, Wannamethee S, Whincup PH. How are falls and fear of falling associated with objectively measured physical activity in a cohort of community-dwelling older men? BMC Geriatr. 2014;14:114.

5. Blum L, Korner-Bitensky N. Usefulness of the Berg Balance Scale in stroke rehabilitation: a systematic review. Phys Ther. 2008;88:559-66.

6. Liphart J, Gallichio J, Tilson JK, Pei Q, Wu SS, Duncan PW: Concordance and discordance between measured and perceived balance and the effect on gait speed and falls following stroke. Clin Rehabil 2015;9:294-302.

7. Weiss A, Brozgol M, Dorfman M, Herman T, Shema S, Giladi N, Hausdorff JM. Does the evaluation of gait quality during daily life provide insight into fall risk? A novel approach using 3-day accelerometer recordings. Neurorehabil Neural Repair. 2013;27:742-52.

8. Rispens SM, van Schooten KS, Pijnappels M, Daffertshofer A, Beek PJ, van Dieën JH: Identification of Fall Risk Predictors in Daily Life Measurements: Gait Characteristics' Reliability and Association With Self-reported Fall History. Neurorehabil Neural Repair. 2014;29:54-61.

9. van Schooten KS, Rispens SM, Elders PJM, Lips P, Pijnappels M, van Dieën $\mathrm{JH}$. Ambulatory fall risk assessment: Quality and quantity of daily-life activities predict falls in older adults. J Gerontol. 2015;70:608-15.

10. Kao PC, Dingwell JB, Higginson JS, Binder-Macleod S. Dynamic instability during post-stroke hemiparetic walking. Gait Posture. 2014;40:457-63.

11. Patterson KK, Parafianowicz I, Danells CJ, Closson V, Verrier MC, Staines WR, Black SE, Mcllroy WE. Gait asymmetry in community-ambulating stroke survivors. Arch Phys Med Rehabil. 2008;89:304-10.

12. Roos M a, Rudolph KS, Reisman DS. The structure of walking activity in people after stroke compared with older adults without disability: a crosssectional study. Phys Ther. 2012;92:1141-7.

13. Folstein MF, McHugh PR, Folstein SE. Mini-mental state. A practical method for grading the cognitive state of patients for the clinician. J Psychiatr Res. 1975;12:189.

14. Lamb SE, JÃ rstad-Stein EC, Hauer K, Becker C: Development of a Common Outcome Data Set for Fall Injury Prevention Trials: The Prevention of Falls Network Europe Consensus. J Am Geriatr Soc 2005, 53:1618-1622.

15. Rispens SM, Pijnappels M, van Schooten KS, Beek PJ, Daffertshofer A, van Dieën $\mathrm{JH}$. Consistency of gait characteristics as determined from acceleration data collected at different trunk locations. Gait Posture. 2014;40:187-92.

16. Punt M, van Alphen B, van de Port IG, van Dieën JH, Michael $K$, Outermans J, Wittink H. Clinimetric properties of a novel feedback device for assessing gait parameters in stroke survivors. J Neuroeng Rehabil. 2014;11:30.

17. Zijlstra W, Hof AL. Assessment of spatio-temporal gait parameters from trunk accelerations during human walking. Gait Posture. 2003;18:1-10.

18. Menz HB, Lord SR, Fitzpatrick RC. Acceleration patterns of the head and pel $v$ is when walking on le v el and irregular surfaces. Gait Posture. 2003;18:35-46. 
19. Lamoth CJC, Beek PJ, Meijer OG. Pelvis-thorax coordination in the transverse plane during gait. Gait Posture. 2002;16:101-14.

20. Weiss A, Sharifi S, Plotnik M, van Vugt JPP, Giladi N, Hausdorff JM. Toward automated, at-home assessment of mobility among patients with Parkinson disease, using a body-worn accelerometer. Neurorehabil Neural Repair. 2011;25:810-8.

21. Punt $M$, Wittink $H$, van der Bent $F$, van Dieën J. Accuracy of estimates of step frequency from a wearable gait monitor. J Mob Technol Med. 2015;4:2-7.

22. Viccaro LJ, Perera S, Studenski SA. Is timed up and go better than gait speed in predicting health, function, and falls in older adults? J Am Geriatr Soc. 2011:59:887-92.

23. Hausdorff JM, Rios DA, Edelberg HK. Gait variability and fall risk in community-living older adults: a 1-year prospective study. Arch Phys Med Rehabil. 2001;82:1050-6.

24. Toebes MJP, Hoozemans MJM, Furrer R, Dekker J, Van Dieën JH. Local dynamic stability and variability of gait are associated with fall history in elderly subjects. Gait Posture. 2012;36:527-31.

25. Doi T, Hirata S, Ono R, Tsutsumimoto K, Misu S, Ando H. The harmonic ratio of trunk acceleration predicts falling among older people: results of a 1-year prospective study. J Neuroeng Rehabil. 2013;10:7.

26. Mackintosh SFH, Hill K, Dodd KJ, Goldie P, Culham E. Falls and injury prevention should be part of every stroke rehabilitation plan. Clin Rehabil. 2005;19:441-51.

Submit your next manuscript to BioMed Central and we will help you at every step:

- We accept pre-submission inquiries

- Our selector tool helps you to find the most relevant journal

- We provide round the clock customer support

- Convenient online submission

- Thorough peer review

- Inclusion in PubMed and all major indexing services

- Maximum visibility for your research

Submit your manuscript at www.biomedcentral.com/submit
Biomed Central 